\title{
DIRECTORY OF IOWA CLAY WORKERS
}

$$
\text { BY }
$$

S. W. BEYER AND I. A. WILLIAMS. 

DIRECTORY OF IOWA CLAY WORKERS.

ADAIR COUNTY

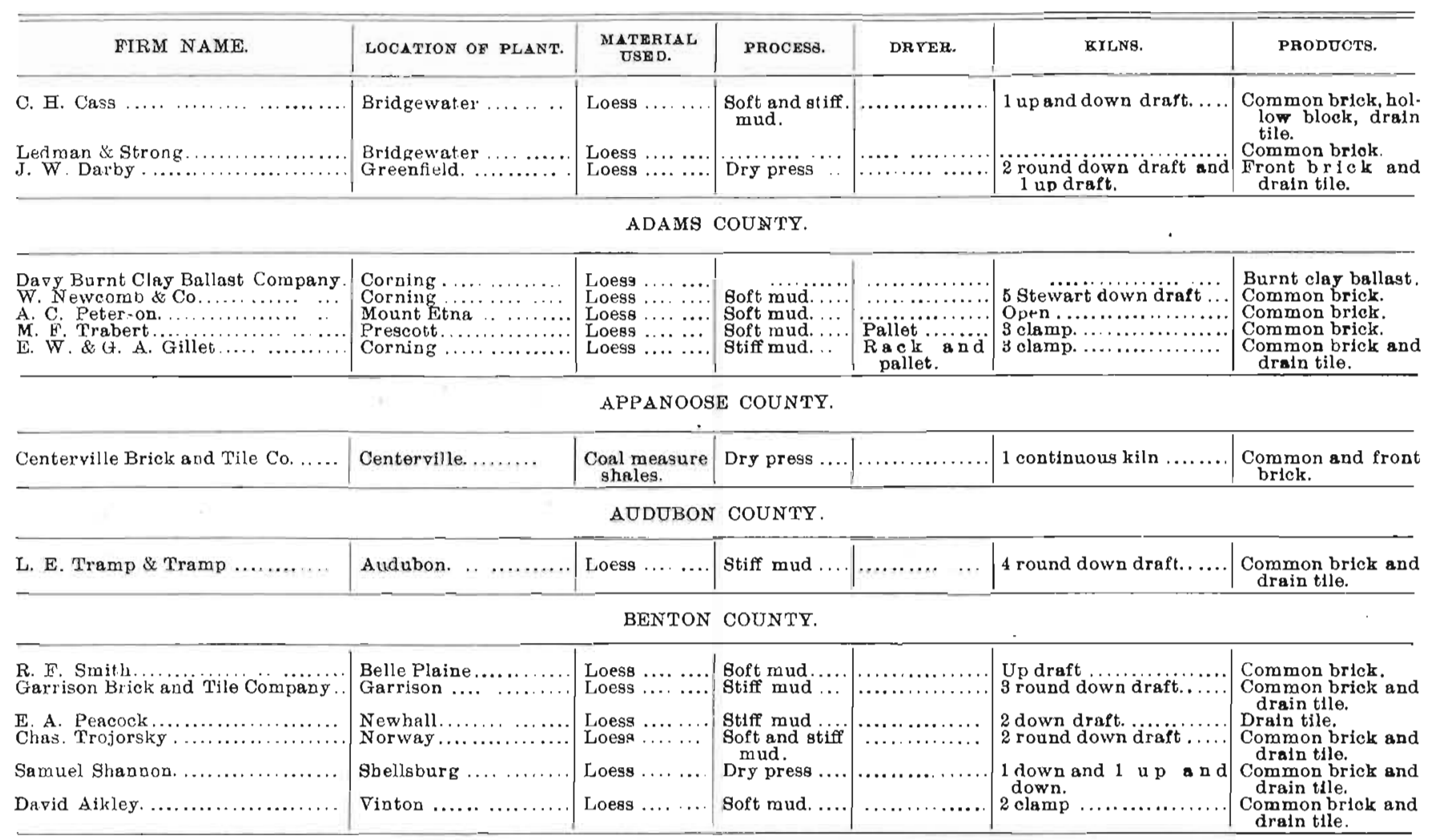


DIRECTORY OF IOW A CLAY WORKERS-CONTINUED.

BLACK BAWK COUNTY.

\begin{tabular}{|c|c|c|c|c|c|c|}
\hline FIRM NAME. & LOCATION OF PLANT. & $\begin{array}{l}\text { MATERIAL } \\
\text { ESED. }\end{array}$ & PROCESS. & DRYER. & KrLNB. & PRODUCTS. \\
\hline $\begin{array}{l}\text { Waterloo \& Cedar Falls Brick Co... } \\
\text { Stead Brothers \& f nonther......... }\end{array}$ & $\begin{array}{l}\text { Cedar Falis ............. } \\
\text { Waterlon } \quad \ldots . . .\end{array}$ & $\begin{array}{l}\text { Kansan and } \\
\text { lowan drift. } \\
\text { Loess }\end{array}$ & $\begin{array}{l}\text { Stiff mud .... } \\
\text { St.iff mnd }\end{array}$ & Phlllips & $\begin{array}{l}\text { Up draft and dow } \\
\text { draft. } \\
\text { 3 romand downdraft..... }\end{array}$ & $\begin{array}{l}\text { Common brick } \\
\text { Common hriek. }\end{array}$ \\
\hline
\end{tabular}

BOONE COUNTY.

\begin{tabular}{|c|c|c|c|c|c|c|}
\hline 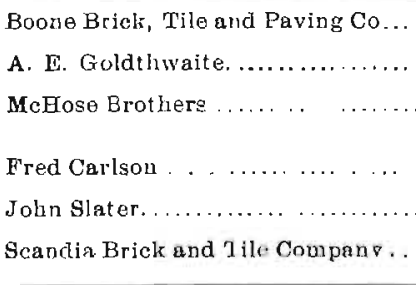 & 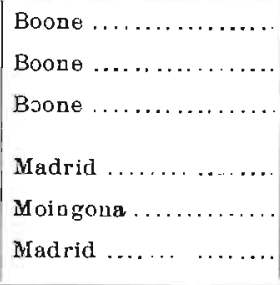 & $\begin{array}{l}\text { Coal measure } \\
\text { shales. } \\
\text { wisconsin } \\
\text { drift. } \\
\text { Shale....... } \\
\text { Drift and } \\
\text { wash. } \\
\text { Drift and } \\
\text { wach. } \\
\text { Shale ......... }\end{array}$ & $\begin{array}{l}\text { Stiff mud } \ldots . \\
\text { Soft mud.... } \\
\text { Stiff mud } \\
\text { Soft mud..... } \\
\text { Soft mud .... } \\
\text { Stiff mud . }\end{array}$ & $\begin{array}{c}\text { Tunnel dryer. } \\
\ldots \ldots \ldots \ldots \ldots \\
\text { Tunnel drser } \\
\ldots \ldots \ldots \ldots \ldots \\
\ldots \ldots \ldots \ldots \ldots \\
\ldots \ldots \ldots \ldots \ldots \\
\ldots \ldots \ldots \ldots\end{array}$ & $\begin{array}{l}10 \text { round down draft.... } \\
\text { Up draft. . ............ } \\
5 \text { round and } 2 \text { square } \\
\text { down draft. } \\
2 \text { up draft, } 1 \text { down draft. } \\
2 \text { up draft, } 1 \text { down draft. } \\
2 \text { round down draft .... }\end{array}$ & $\begin{array}{l}\text { Common brick and } \\
\text { drain tile. } \\
\text { Paving brick and } \\
\text { common brick. } \\
\text { Common brick, } \\
\text { drain tile and } \\
\text { frebrick. } \\
\text { Common brick. } \\
\text { Common brick. } \\
\text { Common brick and } \\
\text { rrain tile. }\end{array}$ \\
\hline
\end{tabular}

BREMER COUNTY

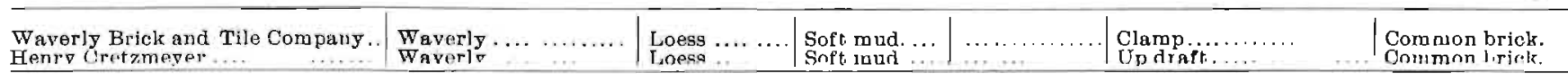

BUEXA VISTA COUNTY.

\begin{tabular}{|c|c|c|c|c|c|c|}
\hline \multicolumn{7}{|c|}{ BUTLER COUNZX. } \\
\hline L. M. Barris \& Bon......... & Clarkville. & rowan drift. & Stiff mud.... & $\ldots$ & Beove No. 2 .. & Comman brick. \\
\hline
\end{tabular}


CALHOUN COUNTT.

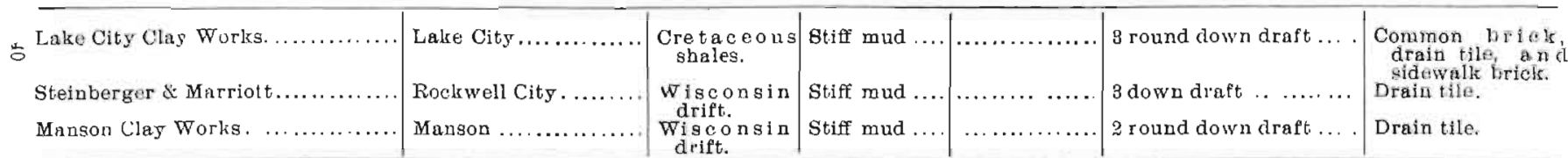

CARROLL COUNTY.

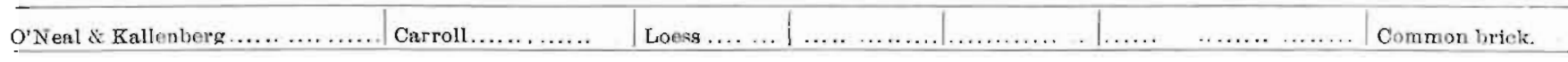

CASB COUNTY.

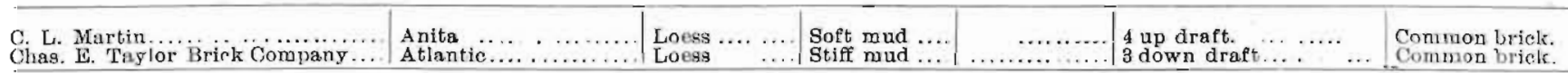

CEDAR COUNTY

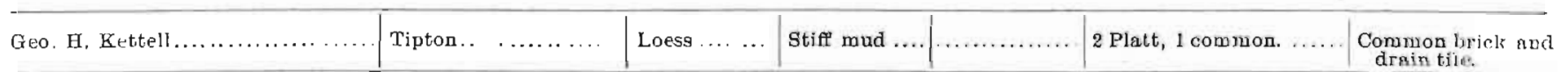

CERRO GORDO COUNTY.

\begin{tabular}{|c|c|c|c|c|c|c|}
\hline Aracrican Bick and Tile Company. & Mason City..... & $\begin{array}{l}\text { Deronian } \\
\text { shales. }\end{array}$ & Stiff mud .... & Tunnel dryer. & 10 down draft. . & $\begin{array}{l}\text { Common brick, } \\
\text { drain tile hollow } \\
\text { block. }\end{array}$ \\
\hline $\begin{array}{l}\text { Mason City Brick and Tile Company } \\
\text { Mason City Clay Worles............. }\end{array}$ & $\begin{array}{l}\text { Macon City... } \\
\text { Mason City..... }\end{array}$ & $\begin{array}{l}\text { Devonian } \\
\text { shales. } \\
\text { Devonian } \\
\text { shales ...... }\end{array}$ & $\begin{array}{l}\text { Stiff mud .... } \\
\text { Stiff mud .... }\end{array}$ & $\begin{array}{l}\text { Tunnel dryer. } \\
\text { Tunnal dryer }\end{array}$ & 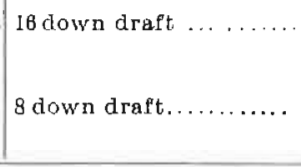 & $\begin{array}{l}\text { Commi on brick, } \\
\text { drain tile, hollow } \\
\text { block. and radial } \\
\text { stack block. } \\
\text { Common brick, } \\
\text { arain tile, hollow } \\
\text { block. }\end{array}$ \\
\hline
\end{tabular}

CHEROKEE COUNTY.

\begin{tabular}{|c|c|c|c|c|c|c|}
\hline own & Cherc & Loess .... .. & Stiff mud: & Iron clad. & $\mid \begin{array}{l}1 \text { down draft, } 3 \text { clamp, } \\
1 \text { continuovs. }\end{array}$ & $\begin{array}{l}\text { Common brick and } \\
\text { drain file. }\end{array}$ \\
\hline
\end{tabular}


CLAX COUNTY.

\begin{tabular}{|c|c|c|c|c|c|c|}
\hline FIRM NAME. & LOUATION OF PLANT. & $\begin{array}{l}\text { MATERTAL } \\
\text { CAED. }\end{array}$ & PROCESS. & DRYER. & KILNS. & PRODUCTS. \\
\hline Hilliard \& Johnson .......... & Spencer.......... & Wiseonsin . & Soft mud... & ...... & 1 up draft 3 down draft. . & Common brick. \\
\hline
\end{tabular}

CLAREE COUNTY

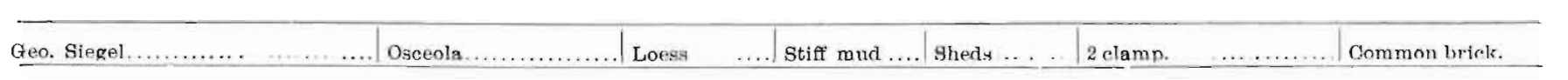

CEATTON COUNTR.

\begin{tabular}{|c|c|c|c|c|c|c|}
\hline 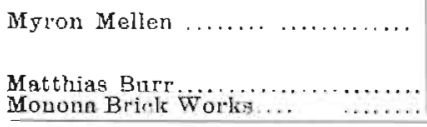 & $\begin{array}{l}\text { Edgewood............... } \\
\text { Guttenberg .......... } \\
\text { Monona........ }\end{array}$ & $\begin{array}{l}\text { Loess and } \\
\text { Maquolreta } \\
\text { shaies. } \\
\text { Loess } \ldots \ldots \\
\text { Loess........ }\end{array}$ & $\begin{array}{l}\text { Stift mud .... } \\
\text { Soft mud. ... } \\
\text { Soft mud .... }\end{array}$ & aheda........ & $\begin{array}{l}1 \text { down draft............ } \\
\text { Clamp. ............ } \\
2 \text { undrafte. downd ruft.. }\end{array}$ & $\begin{array}{l}\text { Common brick and } \\
\text { drain tile. } \\
\text { Common brick. } \\
\text { Common brick. } \\
\end{array}$ \\
\hline
\end{tabular}

\section{CLINTON COUNTY.}

\begin{tabular}{|c|c|c|c|c|c|c|}
\hline 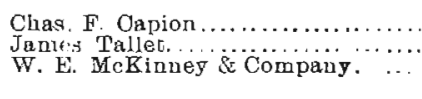 & 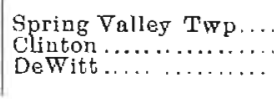 & $\begin{array}{l}\text { Loess. ......... } \\
\text { Loess......... } \\
\text { Loe-g, ....... }\end{array}$ & $\begin{array}{l}\text { Soft mud..... } \\
\text { Soft mud..... } \\
\text { Btift mud.... }\end{array}$ & a............ & 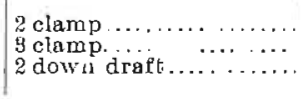 & $\begin{array}{l}\text { Common brick. } \\
\text { Common brick. } \\
\text { Common brick and } \\
\text { drain tile. }\end{array}$ \\
\hline
\end{tabular}

CRAWFORD COUNTY

\begin{tabular}{|c|c|c|c|c|c|c|}
\hline 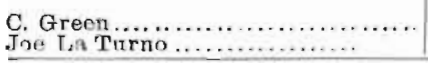 & $\begin{array}{l}\text { Denison .............. } \\
\text { Man1la ............. } \\
\end{array}$ & $\begin{array}{lll}\text { Loess. } & . . & . . . \\
\text { Loess. .......... }\end{array}$ & $\begin{array}{l}\text { Stiff mud. .... } \\
\text { Soft mud .... }\end{array}$ & a...... & $\begin{array}{l}2 \text { clamp, } 1 \text { down draft. } \\
2 \text { no draft.. }\end{array}$ & $\begin{array}{l}\text { Common brick. } \\
\text { Common brisk. }\end{array}$ \\
\hline
\end{tabular}


DALLAS COUNTY

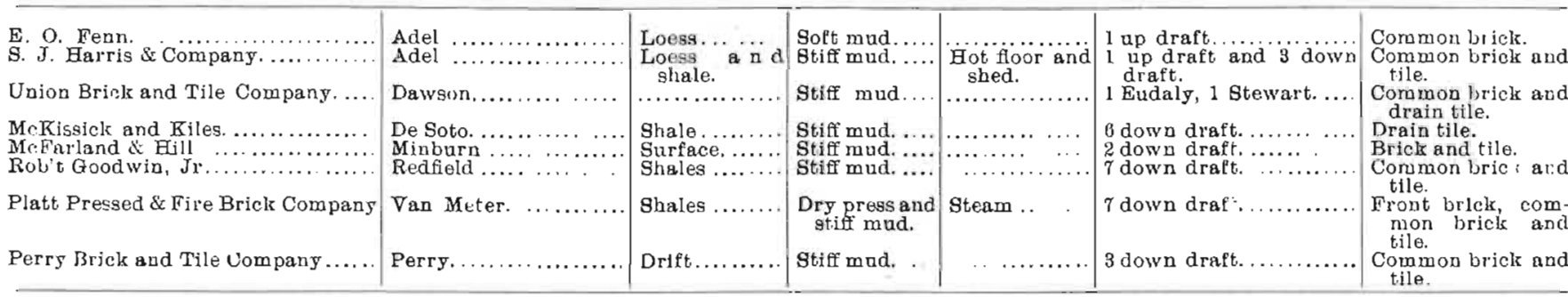

DAVIS COUNTY.

\begin{tabular}{|c|c|c|c|c|c|}
\hline M. L. Parker. & Eldon. & Loess. & Soft mud.... & 1 clamp... & Conmon brick. \\
\hline A. P. Birchmier $\ldots \ldots \ldots \ldots \ldots \ldots$ & Troy............. & Loess..... & Soft mur. & 1 round down draft & Common brick. \\
\hline
\end{tabular}

DECATUR COUNTY.

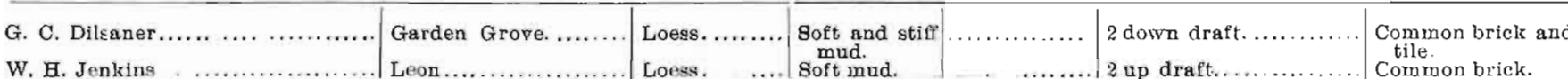

DELAWARE COUNTY.

\begin{tabular}{|c|c|c|c|c|c|c|}
\hline 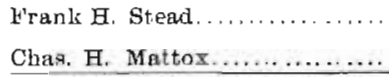 & $\begin{array}{l}\text { Bopkinton ........ } \\
\text { Manchester........ }\end{array}$ & 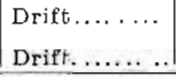 & $\begin{array}{l}\text { Stiff } \operatorname{mud} \ldots \\
\text { Soft mud..... }\end{array}$ & …...... & $\begin{array}{l}2 \text { round down d aft. .... } \\
\text { scove. ....................... }\end{array}$ & $\begin{array}{l}\text { Common and face } \\
\text { brick. } \\
\text { Common brick. }\end{array}$ \\
\hline
\end{tabular}

DES MOINES COUNTY

\begin{tabular}{|c|c|c|c|c|c|c|}
\hline Burli & Burling & & & & 1 dowe draft. & Stoneware. \\
\hline $\begin{array}{l}\text { The Grauite Brick Company.... } \\
\text { Mediopolis Tile and Brick Works }\end{array}$ & $\begin{array}{l}\text { Cascade...... } \\
\text { Mediopolia.... }\end{array}$ & $\begin{array}{c}\text { Kinderliook } \\
\text { shales. } \\
\text { Loess ......... }\end{array}$ & $\begin{array}{c}\text { Stiff mud..... } \\
\text { Stiff mud .... }\end{array}$ & Hot floor.... & $\begin{array}{l}5 \text { Eudaly.......... } \\
3 \text { round down dre }\end{array}$ & $\begin{array}{l}\text { Paving and com- } \\
\text { mon brick and } \\
\text { front brids. } \\
\text { Common brick. }\end{array}$ \\
\hline
\end{tabular}


DIRECTORY OF IOWA CLAY WORKERS-CONTINTED.

\begin{tabular}{|c|c|c|c|c|c|c|}
\hline FIRM NAME. & LOCATION OF PLANT. & $\begin{array}{l}\text { MATBRIAL } \\
\text { TSED. }\end{array}$ & PROCESS. & DRYER. & KILNS. & PRODECTS. \\
\hline 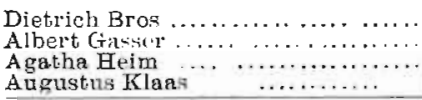 & 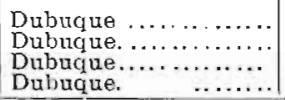 & $\begin{array}{l}\text { Loess } \ldots . . . \\
\text { Loess } \ldots . . . \\
\text { Loess } \ldots . . . . \\
\text { Loess } \ldots . . . . . . \\
\end{array}$ & $\begin{array}{l}\text { Boft mad..... } \\
\text { 8oft mud..... } \\
\text { Boft mud. .... } \\
\text { Soft mud.... }\end{array}$ & 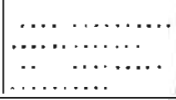 & 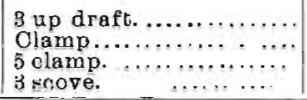 & $\begin{array}{l}\text { Common brick. } \\
\text { Common briekt. } \\
\text { Common brick. } \\
\text { Coramon brick. }\end{array}$ \\
\hline
\end{tabular}

EMMET COUNTY.

\begin{tabular}{|c|c|c|c|c|c|c|}
\hline Armetrong Brick and Tile Company & Armstrong ... & \begin{tabular}{|} 
Wisconsin \\
drift.
\end{tabular} & Stiff paud & Stcam...... & Down draft............... & Drain tile. \\
\hline \multicolumn{7}{|c|}{ FAYETTE COUNTY. } \\
\hline $\begin{array}{l}\text { C. Miller \& Sol } \ldots \ldots \ldots \ldots \\
\text { I. A Book } \ldots \ldots \ldots\end{array}$ & $\begin{array}{l}\text { Clermout...... .... } \\
\text { Bawkese............ }\end{array}$ & $\left|\begin{array}{l}\text { Maquoketa } \\
\text { shales. } \\
\text { Surfaco..... }\end{array}\right|$ & $\begin{array}{l}\text { Stiff mud... } \\
\text { Soft mud. }\end{array}$ & & 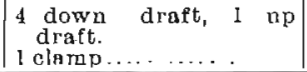 & $\begin{array}{l}\text { Commou brick, tile } \\
\text { and hollow bloek } \\
\text { Common brick. }\end{array}$ \\
\hline
\end{tabular}

FLOYD COUNTX.

\begin{tabular}{|c|c|c|c|c|c|c|}
\hline L. M. Harris & Rockford .............. & $\begin{array}{l}\text { Deronian } \\
\text { shales. }\end{array}$ & Stlff mand ..... & $\ldots$ & 2 soove, 2 down draft... & $\begin{array}{l}\text { Common brick and } \\
\text { tilf. }\end{array}$ \\
\hline
\end{tabular}

FRANELIN COUNTY.

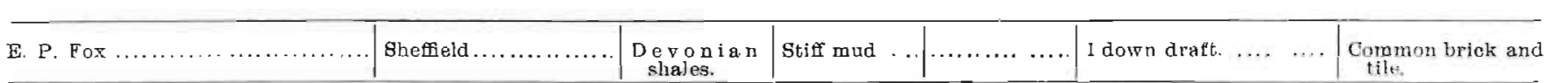

FREMONT COUNTY.

\begin{tabular}{|c|c|c|c|c|c|c|}
\hline 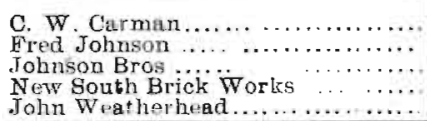 & 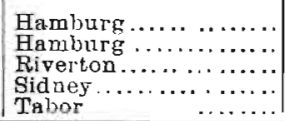 & 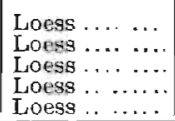 & 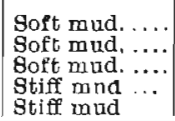 & 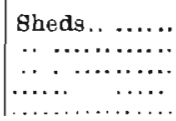 & 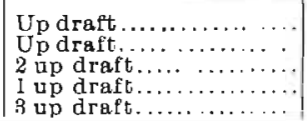 & $\begin{array}{l}\text { Common brick. } \\
\text { Common brick. } \\
\text { Common brick. } \\
\text { Common brick. } \\
\text { Common briels. }\end{array}$ \\
\hline
\end{tabular}


GREENE COUNTY

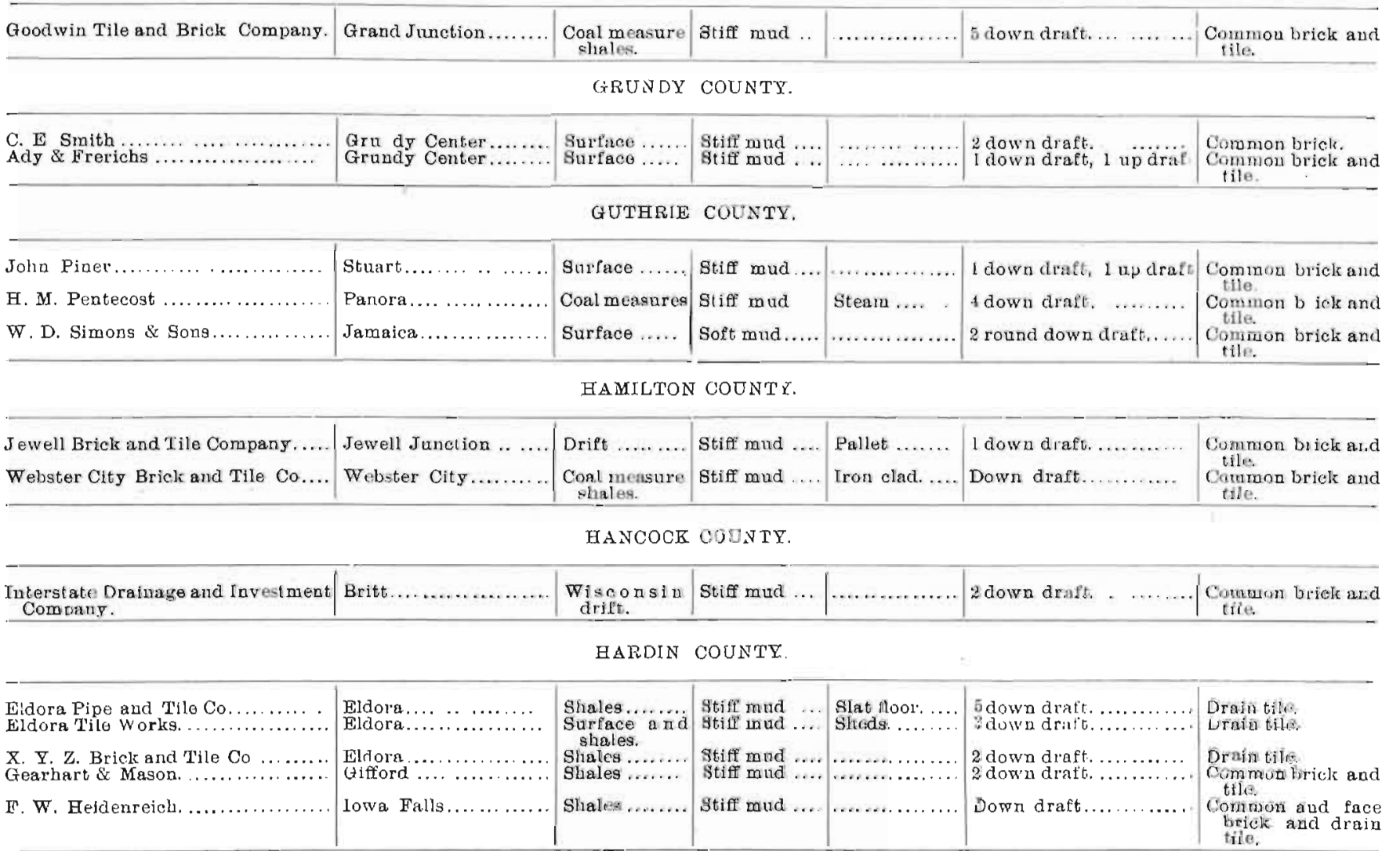


DIRECTORY OF IOWA OLAY WORKERS-OONTINUED.

HARRISON COUNTY

\begin{tabular}{|c|c|c|c|c|c|c|}
\hline 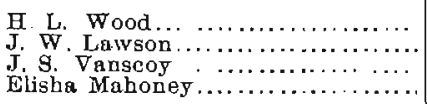 & \multirow{2}{*}{ 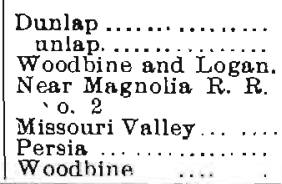 } & $\begin{array}{l}\text { Loess. ....... } \\
\text { Loes. ........ } \\
\text { Loesg. ...... } \\
\text { Loess....... }\end{array}$ & $\begin{array}{l}\text { Soft mud. } \\
\text { Soft mud..... } \\
\text { Soft mud..... } \\
\text { Soft mud..... }\end{array}$ & 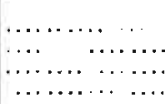 & $\begin{array}{l}\text { qup draft. } \\
\text { Up draft } \ldots \ldots \ldots \ldots \ldots \ldots \ldots \\
\text { Clamp } \ldots \ldots \ldots \ldots \\
\text { Up draft } \ldots \ldots \ldots \ldots \ldots \ldots\end{array}$ & $\begin{array}{l}\text { Common brick. } \\
\text { Common bick. } \\
\text { Common brick. } \\
\text { Common brick. }\end{array}$ \\
\hline $\begin{array}{l}\text { Chas. Smith } \\
\text { M. Analyser } \\
\text { Canfeld \& Crnmio } \ldots \ldots \ldots \ldots \ldots \ldots \ldots \ldots \\
\end{array}$ & & $\begin{array}{l}\text { Loess. ........ } \\
\text { Loess........ } \\
\text { Loess. ....... }\end{array}$ & $\begin{array}{l}\text { Soft mnd..... } \\
\text { Soft mud..... } \\
\text { Soft mud. }\end{array}$ & & $\begin{array}{l}\text { Up draft } \ldots \ldots \ldots \ldots \ldots \ldots \\
\text { Up draft } \ldots \ldots \ldots \ldots \ldots \ldots \ldots \\
3 \text { up draft, } \ldots \ldots \ldots \ldots \ldots\end{array}$ & $\begin{array}{l}\text { Common brick. } \\
\text { Common brick. } \\
\text { Common brick. }\end{array}$ \\
\hline
\end{tabular}

HENRY COUNTY.

\begin{tabular}{|c|c|c|c|c|c|c|}
\hline 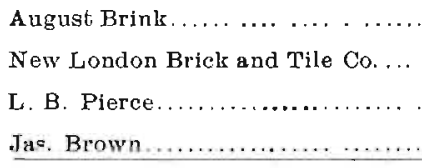 & $\begin{array}{l}21 / 2 \text { miles south of New } \\
\text { London. } \\
\text { New London........... } \\
\text { Winfield.............. } \\
\text { Mount Pleasant. ..... }\end{array}$ & $\begin{array}{l}\text { Loess. . . . . . . } \\
\text { Loess......... } \\
\text { Loess ......... } \\
\text { Loess. ........ }\end{array}$ & $\begin{array}{l}\text { Stiff mud. } \\
\text { Stiff mud. } \\
\text { Stiff mud. } \\
\text { Soft mud. }\end{array}$ & Am. Blower. & 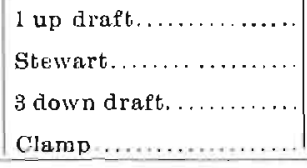 & $\begin{array}{l}\text { Common brick and } \\
\text { drain tile. } \\
\text { Common brick and } \\
\text { tile. } \\
\text { Common brick and } \\
\text { tile. } \\
\text { Common brick. } \\
\end{array}$ \\
\hline
\end{tabular}

HOW ARD COUNTY.

\begin{tabular}{|c|c|c|c|c|c|c|}
\hline Wheeler \& Marshall. ............... & Cresco. & Iowan Drift.. & Stiff mud. & s eds........... & 3. down draft. & $\begin{array}{l}\text { Common brick } \\
\text { drain tile and } \\
\text { hollow block. }\end{array}$ \\
\hline
\end{tabular}

HUMBOLDT COUNTY.

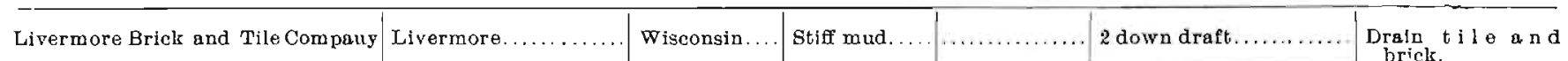

IDA CODNTY.

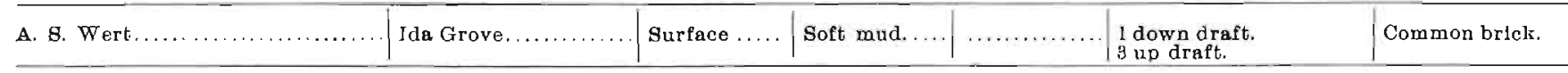


IOWA COUNTY

\begin{tabular}{|c|c|c|c|c|c|c|}
\hline 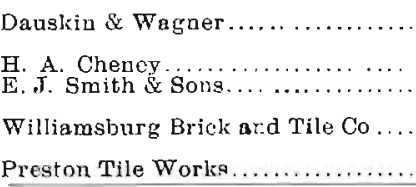 & 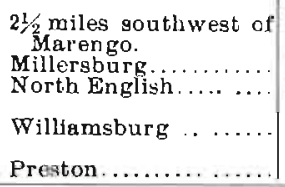 & $\begin{array}{l}\text { Surface ..... } \\
\text { Loess......... } \\
\text { Loess....... } \\
\text { Loess......... } \\
\text { Loess. ........ }\end{array}$ & $\begin{array}{l}\text { Stiff mud.... } \\
\text { Soft mud.... } \\
\text { Stiff mt: ... } \\
\text { Stiff mad..... } \\
\text { Stiff mud..... }\end{array}$ & 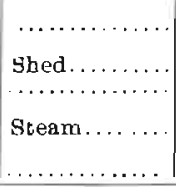 & 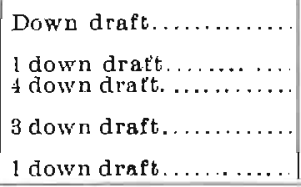 & $\begin{array}{l}\text { Common brick. } \\
\text { Oommon brick, } \\
\text { Common brick and } \\
\text { tile. } \\
\text { Common brick and } \\
\text { tile. } \\
\text { Drain tile. }\end{array}$ \\
\hline \multicolumn{7}{|c|}{ JASPER COUNTY. } \\
\hline 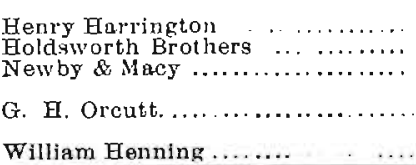 & 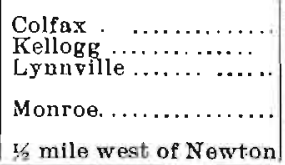 & $\begin{array}{l}\text { Loess } \ldots \ldots \ldots \\
\text { Loess } \ldots \ldots \ldots \\
\text { Loess } \ldots . . . \\
\text { Loess } \ldots \ldots \ldots \\
\text { Loess } \ldots . .\end{array}$ & $\begin{array}{l}\text { Soft mud... } \\
\text { Stiff mud } \ldots . \\
\text { Stiff mud .... } \\
\text { Stiff mud ... } \\
\ldots \ldots\end{array}$ & 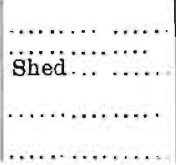 & $\left|\begin{array}{l}3 \text { up draft. ................ } \\
1 \text { down draft, i Bbewart. } \\
3 \text { down draft. ........... } \\
2 \text { up draft, } 2 \text { Cooke...... }\end{array}\right|$ & $\begin{array}{l}\text { Common brick. } \\
\text { Brick and tile. } \\
\text { Common brick and } \\
\text { tile. } \\
\text { Common brick and } \\
\text { drain tile. } \\
\text { Common brick. }\end{array}$ \\
\hline
\end{tabular}

JEFFERSON DOUNTY.

\begin{tabular}{|c|c|c|c|c|c|c|}
\hline 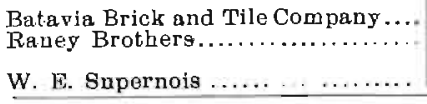 & $\begin{array}{l}\text { Batavia } \ldots \ldots \ldots \ldots \ldots \\
\text { Fairfleld } \ldots \ldots \ldots \ldots \ldots \\
\text { Packwood } \ldots \ldots \ldots \ldots \ldots\end{array}$ & $\begin{array}{l}\text { Loess ......... } \\
\text { Loes s a d d } \\
\text { shales. } \\
\text { Loess ....... }\end{array}$ & $\begin{array}{l}\text { Stiff mud } \ldots \\
\text { Stiff mud ... } \\
\text { Stiff mud }\end{array}$ & 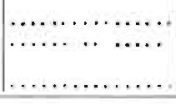 & $\begin{array}{l}1 \text { down draft. ............ } \\
\text { Dowu draft ........... } \\
2 \text { down draft. ... . . . }\end{array}$ & $\begin{array}{l}\text { Brick and tile. } \\
\text { Common brick and } \\
\text { tile. } \\
\text { Drain tile. }\end{array}$ \\
\hline \\
\hline 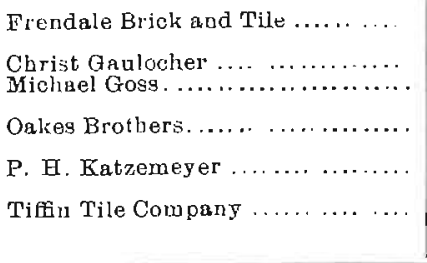 & 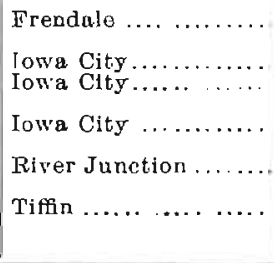 & 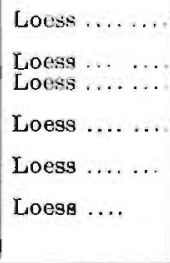 & $\begin{array}{l}\text { Stiff mud .... } \\
\text { Soft mud... } \\
\text { Sof mud.... } \\
\text { Stiff mud .... } \\
\text { Soft mud. ... } \\
\text { Stiff mud .... }\end{array}$ & 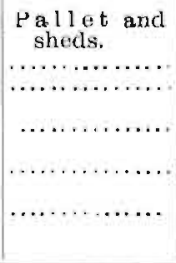 & 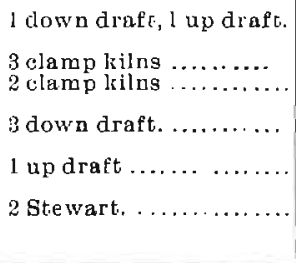 & $\begin{array}{l}\text { Commun brick and } \\
\text { drain tile. } \\
\text { Common brick. } \\
\text { Common aud front } \\
\text { brick. } \\
\text { Common brick and } \\
\text { drain tile. } \\
\text { Common and front } \\
\text { brick. } \\
\text { Comm on brick, } \\
\text { drain tile and } \\
\text { hollow block. }\end{array}$ \\
\hline
\end{tabular}

JONES COUNTY.

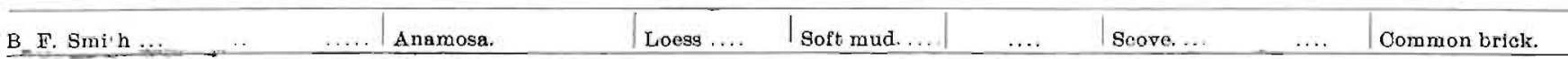


D IRECTORY OF IOWA CLAY WORKERS-CONTYNEED

KEORUK OOUNTY

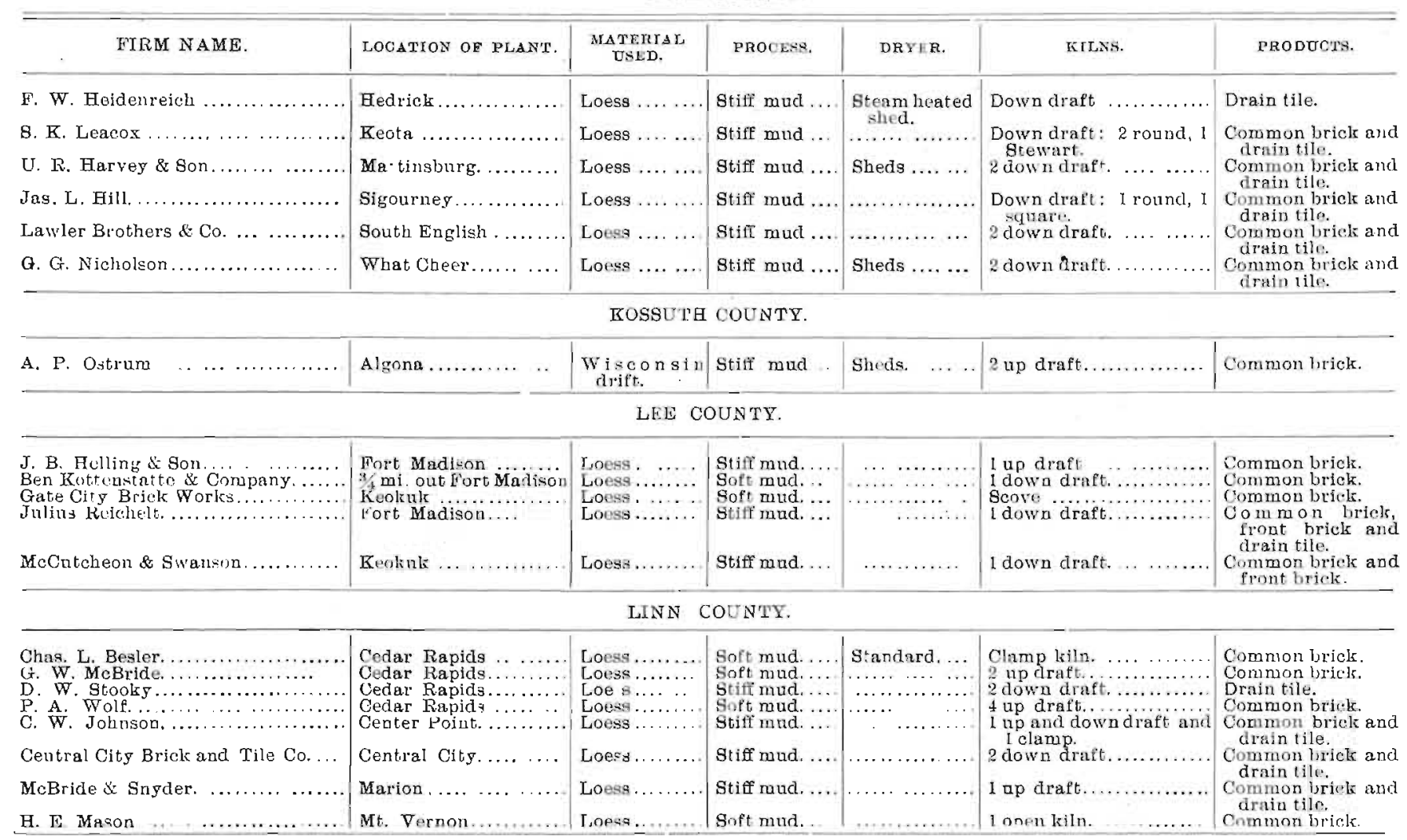


LOUISA COUNTY.

\begin{tabular}{|c|c|c|c|c|c|c|}
\hline $\begin{array}{l}\text { G. W. Oakes.............................. } \\
\text { McClurkin, Ochiltreo \& Compang. }\end{array}$ & $\begin{array}{l}\text { Columbus Junction ... } \\
\text { Morning Suu......... }\end{array}$ & $\begin{array}{c}\text { Loess.......... } \\
\text { Loess......... }\end{array}$ & $\begin{array}{l}\text { Stiff mud... } \\
\text { stiff mucl.... }\end{array}$ & & $\begin{array}{l}\ldots \ldots \ldots \ldots \\
1 \text { Stewart........ }\end{array}$ & $\begin{array}{l}\text { Common brick and } \\
\text { drain tile. } \\
\text { Common brick and } \\
\text { druin tile. }\end{array}$ \\
\hline
\end{tabular}

LUCAS COUNTY.

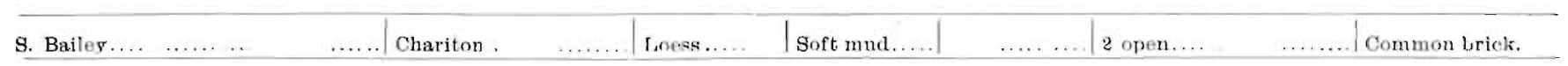

MADISON COUNTY.

\begin{tabular}{|c|c|c|c|c|c|c|}
\hline Clark Brog. ......................... & Winterset $\ldots . . . \cdots \ldots$ & Loess ....... .. & $\begin{array}{l}\text { Soft and stiff } \\
\text { mud. }\end{array}$ & $\ldots$ & 2 down draft. ............. & $\begin{array}{l}\text { Common brick and } \\
\text { drain tile. }\end{array}$ \\
\hline \multicolumn{7}{|c|}{ MAHASEA COUNTY. } \\
\hline 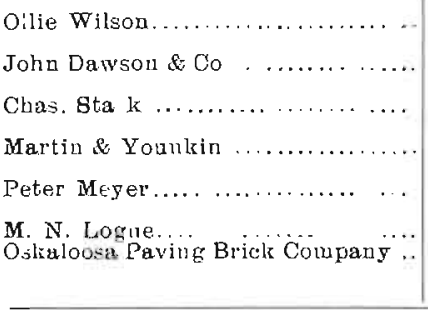 & 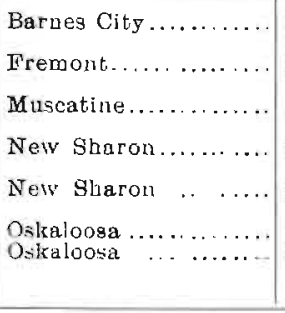 & 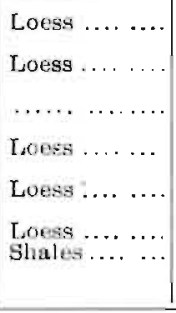 & $\begin{array}{l}\text { Stiff mud } \ldots \\
\text { Stiff mud } \ldots . \\
\ldots \ldots \ldots \\
\text { Stiff mud } \ldots \\
\text { Stiff mud ..... } \\
\text { Soft mud ... } \\
\text { Silf mud }\end{array}$ & $\begin{array}{l}\text { Sheds......... } \\
\ldots \ldots \ldots \ldots . . . . \\
\ldots \ldots \ldots \ldots . \\
\text { Steam ....... } \\
\ldots \ldots \ldots \ldots .\end{array}$ & 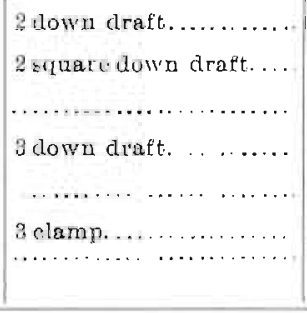 & 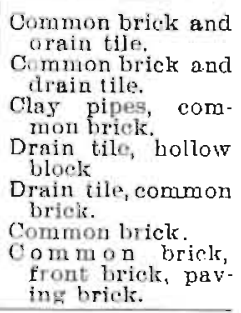 \\
\hline
\end{tabular}

MARION COUNTY.

\begin{tabular}{|c|c|c|c|c|c|c|}
\hline Elog Bros. & Knoxvilie...... & I Loess .... : & Stiff mud. & & 1 down draft. & ick and \\
\hline 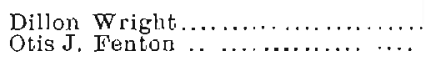 & $\begin{array}{l}\text { Knoxville........... } \\
\text { Otterbeill......... }\end{array}$ & 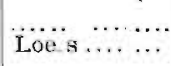 & Stiff mud & $\cdots \cdots$ & i elamp.... & Common brick and \\
\hline Pella Drain Tile Company.......... & Pella... & Loesa ....... & Stiff mua & & 5own draft... & $\begin{array}{l}\text { Oonmon brick and } \\
\text { drain tile. }\end{array}$ \\
\hline
\end{tabular}


MARSHAIL COUNTY

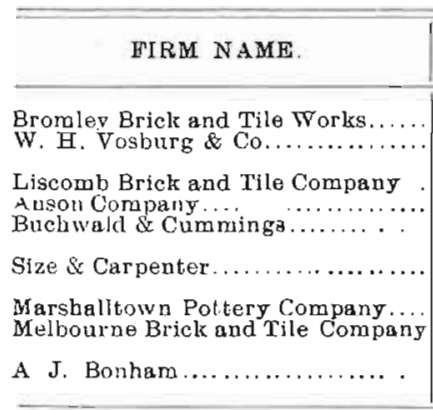

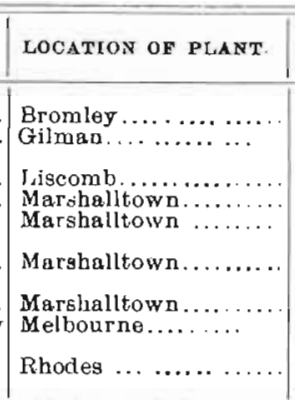

\begin{tabular}{|c|c|c|}
\hline $\begin{array}{l}\text { MATERIAL } \\
\text { USED. }\end{array}$ & PROCESS. & DRYER. \\
\hline $\begin{array}{l}\text { Loess ......... } \\
\text { Loess .... ... }\end{array}$ & $\begin{array}{l}\text { Stiff mud . } \\
\text { Stiff } m a d \ldots\end{array}$ & \\
\hline $\begin{array}{l}\text { Loess } . . . . . . . \\
\text { Loess } . . . . . . \\
\text { Loe:s .... .... }\end{array}$ & $\begin{array}{l}\text { Suiff mud .... } \\
\text { Dry press .... } \\
\text { Stiff mud ... }\end{array}$ & ……....... \\
\hline Loess ........ & $\begin{array}{l}\text { soft and stiff } \\
\text { mud. }\end{array}$ & $\begin{array}{l}\text { Rack and pr } \\
\text { let. }\end{array}$ \\
\hline 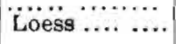 & sitiof roud .... & \\
\hline Loess ........ & Stiff mud ... & \\
\hline
\end{tabular}

\begin{tabular}{|c|c|}
\hline KILNG. & \\
\hline $\begin{array}{l}3 \text { down draft........... } \\
1 \text { up draft, } 1 \text { downd draft. }\end{array}$ & \\
\hline $\begin{array}{l}5 \text { down draft............ } \\
3 \text { updraft. } \ldots \ldots \ldots \ldots \ldots \\
2 \text { down draft clump...... }\end{array}$ & \\
\hline 6 updraft........ & \\
\hline $\begin{array}{l}4 \text { down draft. } \ldots \ldots \ldots \\
1 \text { down draft, } z \text { gtewart }\end{array}$ & \\
\hline 2 down draft. & \\
\hline
\end{tabular}

PRODUCTs.

MILLS OOUNTY.

\begin{tabular}{|c|c|c|c|c|c|c|}
\hline 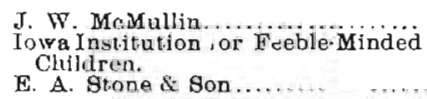 & 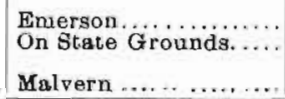 & $\mid \begin{array}{l}\text { Loess } \ldots . . . . \\
\text { Loess } \ldots . . . . \\
\text { Loess } \ldots . . .\end{array}$ & $\begin{array}{l}\text { Soft mud..... } \\
\text { Soft mud... } \\
\text { Stiff mud }\end{array}$ & $\mid \begin{array}{c}\ldots \ldots \ldots \ldots \ldots \\
\ldots \ldots \ldots \ldots \\
\text { Sheda } \ldots \ldots \ldots\end{array}$ & 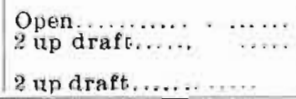 & $\begin{array}{l}\text { Common brick. } \\
\text { Common brick. } \\
\text { Common brick. }\end{array}$ \\
\hline
\end{tabular}

MONONA COYNTY

$$
\text { 然 }
$$

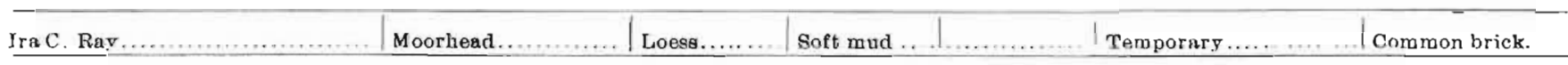

MONROE COUNTY.

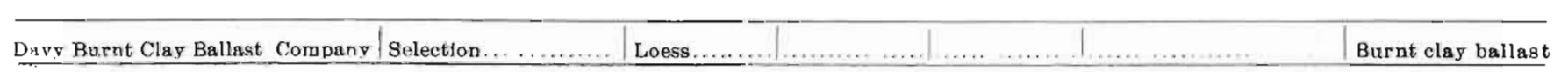


MONTGOMERY COUNTY.

\begin{tabular}{|c|c|c|c|c|c|c|}
\hline 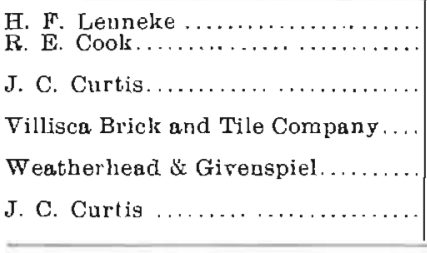 & $\begin{array}{l}\text { Elliotit....... } \\
\text { Red Oak.... } \\
\text { Red Oak.... } \\
\text { Villisca..... } \\
\text { Red Oak.... } \\
\text { Red Oak.... }\end{array}$ & $\begin{array}{l}\text { Loess ......... } \\
\text { Locss. ....... } \\
\text { L o e s g and } \\
\text { shale. } \\
\text { Loess......... } \\
\text { Loess. ........ } \\
\text { Shale......... }\end{array}$ & $\begin{array}{l}\text { Soft mud ... } \\
\text { Stiff mud .... } \\
\text { s t i ff m u d, } \\
\text { dry press. } \\
\text { Stiff mud .... } \\
\text { Soft mud and } \\
\text { dry presy. }\end{array}$ & Bucgrus. & 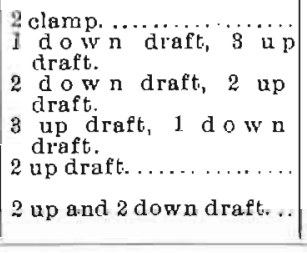 & $\begin{array}{l}\text { Common brick, } \\
\text { Common brick and } \\
\text { drain tile. } \\
\text { Common, front and } \\
\text { firebrick. } \\
\text { Common brick and } \\
\text { drain tile. } \\
\text { Common, pressed } \\
\text { brick. } \\
\text { Red earthenware } \\
\text { and stoneware. }\end{array}$ \\
\hline 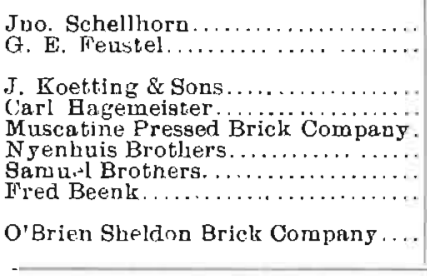 & $\begin{array}{l}\text { Fairport...... } \\
\text { F'airport...... } \\
\text { Muscatine.... } \\
\text { Muscatine.... } \\
\text { Museatine.... } \\
\text { Muscatine.... } \\
\text { Muscatine... } \\
\text { Wheatland... } \\
\text { Sheldon........ }\end{array}$ & 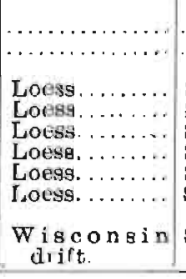 & $\begin{array}{l}\text { Soft mud.... } \\
\text { Soft mud.... } \\
\text { Soft mud. } \\
\text { Soft mud.... } \\
\text { Soft mud..... } \\
\text { Siff mud.... } \\
\text { Soft mud..... }\end{array}$ & & 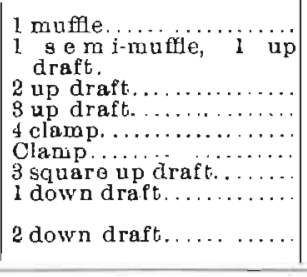 & $\begin{array}{l}\text { Red earthenware. } \\
\text { Red earthenware. } \\
\text { Common brick. } \\
\text { Commont brick. } \\
\text { Conmon brick. } \\
\text { Common brick. } \\
\text { Common briok. } \\
\text { Common brick and } \\
\text { draiu tile. } \\
\text { Common brick. }\end{array}$ \\
\hline \multicolumn{7}{|c|}{ PAGE COUNTY. } \\
\hline \multicolumn{7}{|c|}{ PLYMOUTE COUNTY. } \\
\hline Jobn A. Leonard & Le Mars .............. & Loess .... & Soft mond & ............... & 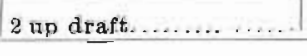 & Common brick. \\
\hline \multicolumn{7}{|c|}{ POCABONTAS COUNTY. } \\
\hline $\begin{array}{l}\text { Straight Bros.... . . . . . . . . . . . . . } \\
\text { Nelson \& Dawson ............ }\end{array}$ & 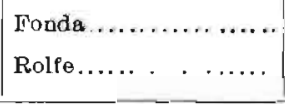 & $\begin{array}{c}\text { Wisconsin } \\
\text { drift. } \\
\text { W I geo in } \\
\text { drift. }\end{array}$ & $\begin{array}{l}\text { Stiff mud... } \\
\text { Stiff mud.... }\end{array}$ & $\mid \begin{array}{l}\cdots \\
\ldots\end{array} \ldots \cdots \cdots$ & $\begin{array}{l}5 \text { down draft. } \ldots \ldots \ldots \ldots \\
2 \text { down draft. .................. }\end{array}$ & $\begin{array}{l}\text { Drain tile. } \\
\text { Drain tile. }\end{array}$ \\
\hline
\end{tabular}


DIRECTORY OF IOW A CLAY WORKERS-CONTINUED.

\begin{tabular}{|c|c|c|c|c|c|c|}
\hline FIRM NAME. & OCATION OE PLANT. & $\operatorname{Max}_{\mathrm{T}}$ & 800 & DHYER, & TLNS. & PRODUCTS. \\
\hline es Moines Co-operative Bri & Moines & odified loess & Soft mud... & & $n$ & Comme \\
\hline ridge Bros ...................... & $n t \ldots \ldots \ldots$ & dioess & Stiti & & I & \\
\hline aving dom & 5 & Shale & Stiff mud & Direct heat. & s. 1 long, i con & $\begin{array}{l}\text { Common } \\
\text { |aving } 1\end{array}$ \\
\hline ale Bricls Company... & Des Moines..... & $\begin{array}{l}\text { Shale and } \\
\text { loess. }\end{array}$ & $\begin{array}{l}\text { Drypress, } \\
\text { stiff mult. }\end{array}$ & Wolff. & 4 clamp, 4 down draft... & $\begin{array}{l}\text { on briek, } \\
t \text { briek and }\end{array}$ \\
\hline $\begin{array}{l}\text { T. Fredregill } \\
\text { owa Bricis Manufacturiu }\end{array}$ & $\begin{array}{l}\text { Des Mc } \\
\text { Des Mc }\end{array}$ & $\begin{array}{l}\text { Modified loess } \\
\text { Sbale....... }\end{array}$ & $\begin{array}{l}\text { Soft n } \\
\text { Stiff I }\end{array}$ & $\begin{array}{l}\text { Sheds ... } \\
\text { Standard. }\end{array}$ & $\begin{array}{l}\text { draft. ............. } \\
e_{1}, 1 \text { round down }\end{array}$ & $\begin{array}{l}n \text { bolk. } \\
\text { brink. } \\
\text { brick ani }\end{array}$ \\
\hline wa Pipe and Tile $\mathrm{C}$ & Des & Shale. & Stif & Siat & & eck. and \\
\hline Likes Im & Des B & Shale........ & Stief mnd & & 8 down $\mathrm{dr}$ & id par- \\
\hline Company .............. & $\begin{array}{l}\text { Des Moines.. } \\
\text { Des Moines.. }\end{array}$ & $\begin{array}{l}\text { Modified loess } \\
\text { Shiale........ }\end{array}$ & $\begin{array}{l}\text { Soft mud. } \\
\text { Stiff mud }\end{array}$ & & $\begin{array}{l}4 \text { down draft. } 2 \text { clanu } p . \\
5 \text { down draft ................ }\end{array}$ & and \\
\hline 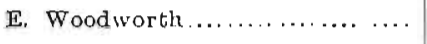 & & & Stiff & & 2 up draft, 1 down & Common brick. \\
\hline$\ldots \ldots \ldots \ldots$ & $\begin{array}{l}\text { Polk } \\
\text { Des } \\
\text { Des D }\end{array}$ & $\begin{array}{l}\text { Surp } \\
\text { Shal } \\
\text { Shal }\end{array}$ & $\begin{array}{l}\text { Stif } \\
\text { Stiff } \\
\text { Stijl }\end{array}$ & Tu & $\begin{array}{ll}2 \mathrm{~d} \\
\mathrm{RO}\end{array}$ & $\begin{array}{l}\mathrm{Dr} \\
\mathrm{Dr} \\
\mathrm{Dr}\end{array}$ \\
\hline
\end{tabular}


POTTAWATTAMIE COUNTY.

\begin{tabular}{|c|c|c|c|c|c|c|}
\hline 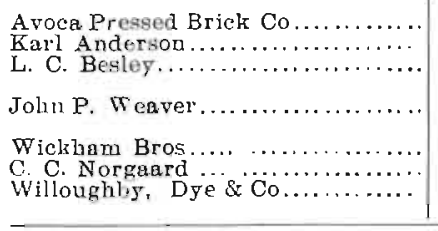 & 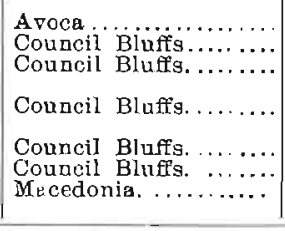 & 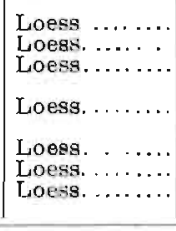 & $\begin{array}{l}\text { Soft mud.... } \\
\text { Soft mud.... } \\
\text { Stiff and soft } \\
\text { mud. } \\
\text { Soft and stiff } \\
\text { mud, } \\
\text { Soft. mud.... } \\
\text { Soft mud..... } \\
\text { Stiff mud... }\end{array}$ & 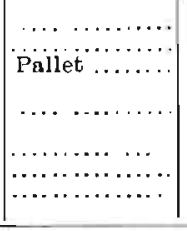 & 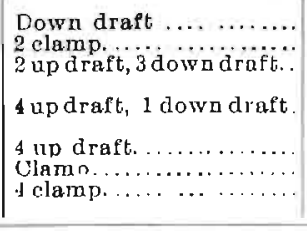 & $\begin{array}{l}\text { Common brick. } \\
\text { Common briclk. } \\
\text { Common brick, } \\
\text { Common bri els, } \\
\text { p4ving brick. } \\
\text { Common brick. } \\
\text { Common brick. } \\
\text { Common brick, } \\
\text { llrain tile }\end{array}$ \\
\hline \multicolumn{7}{|c|}{ POWESAIEK COUNTX. } \\
\hline $\begin{array}{l}\text { Me Eose Bros.... } \\
\text { B. J. Broaditon }\end{array}$ & $\begin{array}{l}\text { Grinnell ............. } \\
\text { Montezuma } \ldots \ldots \ldots \ldots\end{array}$ & $\begin{array}{l}\text { Loess. ......... } \\
\text { Loess. .......... }\end{array}$ & $\begin{array}{l}\text { Stiff mud..... } \\
\text { Stiff mud..... }\end{array}$ & $\ldots \ldots \ldots$ & $\begin{array}{l}3 \text { down draft. ........... } \\
\text { Down draft .............. }\end{array}$ & $\begin{array}{l}\text { Common brick, } \\
\text { drain tile, hollow } \\
\text { block. } \\
\text { Common brick, } \\
\text { drain tile. }\end{array}$ \\
\hline \multicolumn{7}{|c|}{ RINGGGOLD COUNTY. } \\
\hline $\begin{array}{l}\text { Diagonal Brick and Ti'e Co......... } \\
\text { K llerton Brirlk and Tile }\end{array}$ & $\left|\begin{array}{l}\text { Diagonal ............... } \\
\text { Kellerton }\end{array}\right|$ & \begin{tabular}{|l|} 
Loess......... \\
Lones.......
\end{tabular} & $\mid \begin{array}{l}\text { Stiff mud. ... } \\
\mathbf{S} \text { iff mud. .... }\end{array}$ & $\mid \begin{array}{l}\text { Shed........... } \\
\text { Sheds ....... }\end{array}$ & $\mid \begin{array}{l}2 \text { up drnet. } \\
\text { l u d draft, } 2 \text { down draft. }\end{array}$ & $\begin{array}{l}\text { Common brick. } \\
\text { Common briek. }\end{array}$ \\
\hline \multicolumn{7}{|c|}{ SAC COUNTY. } \\
\hline 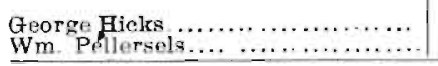 & $\mid \begin{array}{l}\text { Grant City } \ldots \ldots \ldots \ldots \\
\text { Sac City } \ldots \ldots \ldots \ldots \ldots\end{array}$ & \begin{tabular}{|l|} 
Loess. ......... \\
Loegs.........
\end{tabular} & $\begin{array}{l}\text { Soft mud. ... } \\
\text { Soft mitd } \ldots .\end{array}$ & $\mid \ldots \ldots \ldots \ldots \ldots$ & 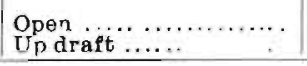 & $\begin{array}{l}\text { Common brick. } \\
\text { Common bricks }\end{array}$ \\
\hline \multicolumn{7}{|c|}{ SCOTT COUNTY. } \\
\hline $\begin{array}{l}\text { Bettendorf Brick and Tile Oo } \\
\text { Davenport Paving Brick and Tile do }\end{array}$ & $\mid \begin{array}{l}\text { Bettendorf ........... } \\
\text { Buffalo ................. }\end{array}$ & 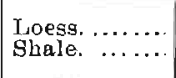 & $\begin{array}{l}\text { Soft mud. .... } \\
\text { Stiff mand..... }\end{array}$ & & $\left|\begin{array}{l}2 \text { open } \ldots \ldots \ldots \ldots \ldots \\
8 \text { down draft. } \ldots \ldots \ldots \ldots\end{array}\right|$ & $\begin{array}{l}\text { Cormmon brick. } \\
\text { Paving briek, drain } \\
\text { tile, hollow block. }\end{array}$ \\
\hline $\begin{array}{l}\text { Black Hawlk Brick Co ............... } \\
\text { Otten \& Pohlmann .................. } \\
\text { H. D. Pohlmann's Sorls.... }\end{array}$ & \begin{tabular}{|l|l|} 
Davenport & $\ldots \ldots \ldots \ldots$ \\
Davenport & $\cdots \cdots \cdots \cdots \cdots$ \\
Davenp rt
\end{tabular} & $\begin{array}{l}\text { Loess. ......... } \\
\text { Loess. .......... } \\
\text { Loess. }\end{array}$ & $\begin{array}{l}\text { Soft mud..... } \\
\text { Soft mud.... } \\
\text { Soft mur... }\end{array}$ & & $\left|\begin{array}{c}4 \operatorname{clamp} \ldots \ldots \ldots \ldots \ldots \ldots \\
4 \operatorname{clamp} \ldots \ldots \ldots \ldots \ldots \ldots\end{array}\right|$ & $\begin{array}{l}\text { Common hrick. } \\
\text { Common brick. } \\
\text { Common brick. } \\
\end{array}$ \\
\hline \multicolumn{7}{|c|}{ SHELBY COUNTY. } \\
\hline Harlan Brick and Tile Company.... & Harlan .. & Loess. ........ & Soft mud.... & $\ldots \quad \ldots$. & 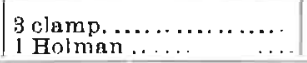 & $\begin{array}{l}\text { Common brick and } \\
\text { drain tile. }\end{array}$ \\
\hline
\end{tabular}


DIRECTORY OF IOWA CLAY WORKERS-CONMINUED.

SIOUX COUITY.

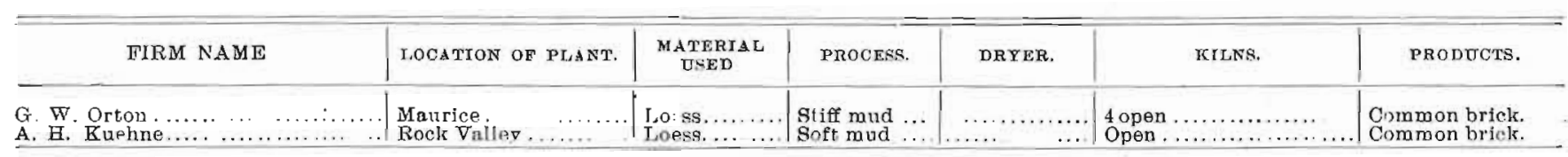

STORY COUNTY

\begin{tabular}{|c|c|c|c|c|c|c|}
\hline 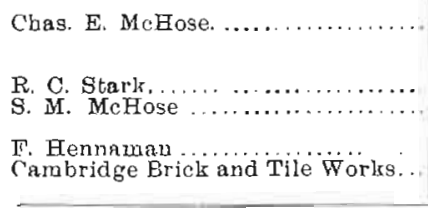 & 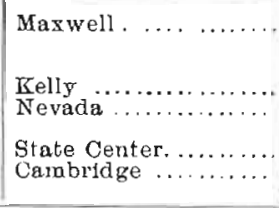 & $\begin{array}{l}\text { Shale ....... } \\
\text { Loess. ........ } \\
\text { Surface .... } \\
\text { Loess. . .... } \\
\text { Loess. .... .. }\end{array}$ & $\begin{array}{l}\text { Stiff mud .... } \\
\text { Sifff mud ... } \\
\text { Stiff mud ... } \\
\text { Soft mud.... } \\
\text { Stiff mud .... }\end{array}$ & 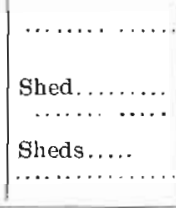 & 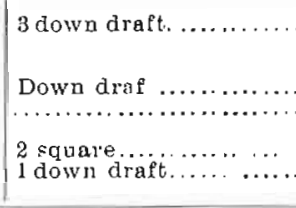 & $\begin{array}{l}\text { Common brick, } \\
\text { paving brick and } \\
\text { drain tile. } \\
\text { Common brick. } \\
\text { Common brick and } \\
\text { drain tile. } \\
\text { Common brick. } \\
\text { Common brick ard } \\
\text { drain tile. }\end{array}$ \\
\hline
\end{tabular}

TAMA COUNTY.

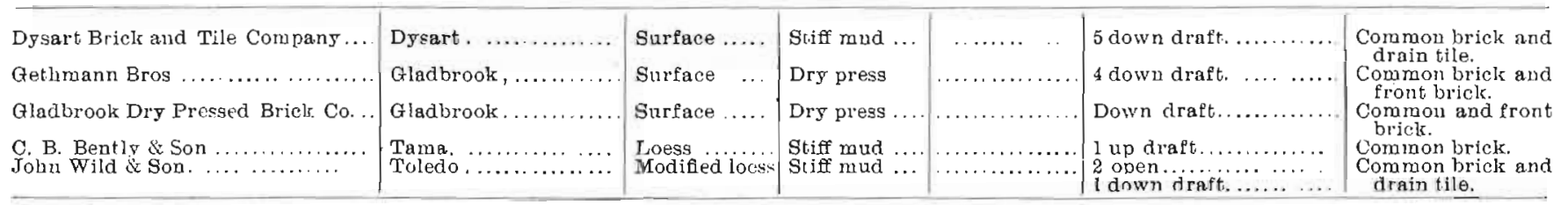

TAYLOR COUNTY.

\begin{tabular}{|c|c|c|c|c|c|c|}
\hline 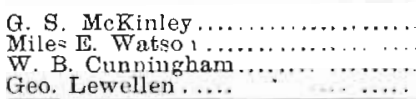 & 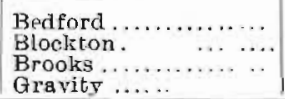 & $\begin{array}{l}\text { Surface ..... } \\
\text { Surface...... } \\
\text { Surface...... } \\
\text { Loes......... }\end{array}$ & $\begin{array}{l}\text { Soft mud } . . . \\
\text { Soft mud.... } \\
\text { Soft mud.... } \\
\text { Soft mud.... }\end{array}$ & $\left|\begin{array}{r}\ldots \ldots \ldots \ldots \\
\cdots \cdots \\
\ldots \ldots \ldots \\
\ldots \ldots \ldots\end{array}\right|$ & 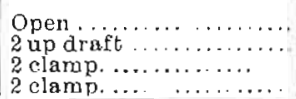 & $\begin{array}{l}\text { Common brick. } \\
\text { Common briek: } \\
\text { Common brick. } \\
\text { Common brick. }\end{array}$ \\
\hline
\end{tabular}


UNION COUNTY.

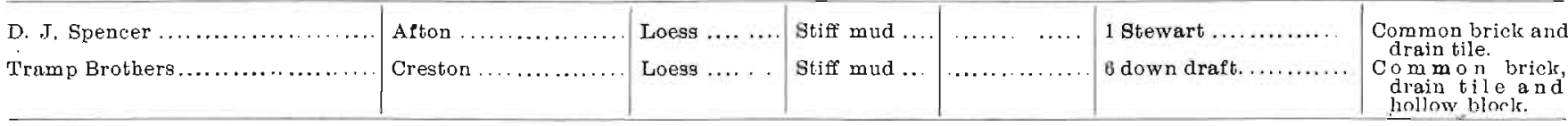

VAN BUREN COJNTY

\begin{tabular}{|c|c|c|c|c|c|c|}
\hline 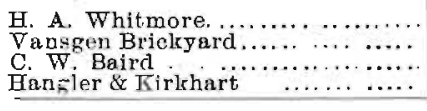 & 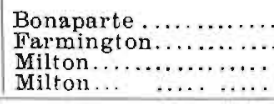 & \begin{tabular}{|l|} 
Surface $\ldots . .$. \\
Surface $\ldots$. \\
Surface $\ldots . .$. \\
Surface $\ldots . .$.
\end{tabular} & $\begin{array}{l}\text { Stiff mud .. } \\
\text { goof mud ... } \\
\text { Stiff mad .. }\end{array}$ & 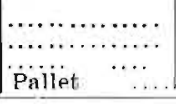 & $\begin{array}{l}\text { Open } \ldots \ldots \\
\text { Open } \ldots \ldots \ldots \\
\text { lup draft } \ldots \ldots \ldots \ldots \\
\text { Down draf }\end{array}$ & $\begin{array}{l}\text { Common brick. } \\
\text { Common brick. } \\
\text { Common brick. } \\
\text { Common brick. }\end{array}$ \\
\hline \multicolumn{7}{|c|}{ WAPELLO COUNTY. } \\
\hline $\begin{array}{l}\text { H. B. Ostdiek } \ldots \ldots \ldots \ldots \ldots \ldots \ldots \\
\text { Ottumwa Brick and Construction } \\
\text { Company. } \\
\text { Swift \& Campbell Brick Oompanr... }\end{array}$ & $\begin{array}{l}\text { Ottumwa. } \\
\text { Ottumwa. }\end{array}$ & $\begin{array}{l}\text { Burface ..... } \\
\text { Shale ....... }\end{array}$ & $\begin{array}{l}\text { Soft mud.... } \\
\text { Stiff mud .... } \\
\text { Stiff mad }\end{array}$ & $\begin{array}{l}\text { Steam. } \\
\text { Sbeds }\end{array}$ & $\begin{array}{l}3 \text { Swift. .................. } \\
1 \text { continuous, } 5 \text { down } \\
\text { draft. } \\
4 \text { up draft, } 1 \text { continnous. }\end{array}$ & $\begin{array}{l}\text { Common brick and } \\
\text { front brick, } \\
\text { Com von briclapay- } \\
\text { ing brick, drain } \\
\text { tille a d bollow } \\
\text { block. } \\
\text { Conmon brink. } \\
\end{array}$ \\
\hline
\end{tabular}

WARREN COUNTY.

\begin{tabular}{|c|c|c|c|c|c|c|}
\hline W. A. Kurtz..... & gartford....... & $\ldots .$. & 1 & $\ldots \ldots$ & Opdraft & Oommon brick. \\
\hline \multicolumn{7}{|c|}{ WAYNE COUNTX. } \\
\hline
\end{tabular}


WEBSTER COUNTY

\begin{tabular}{|c|c|c|c|c|c|c|}
\hline FIRM NAME. & LOCATION OF PLANT. & $\begin{array}{l}\text { MATERIAL } \\
\text { USED. }\end{array}$ & PROCYRS. & DBYER. & ISILNS. & PRODUCTS. \\
\hline $\begin{array}{l}\text { For Dorgo St meware Company . } \\
\text { Bradshaw } x \text { ('ompany................ }\end{array}$ & $\begin{array}{l}\text { Fort Dodge .... } \\
\text { Fort Dodge.... }\end{array}$ & $\begin{array}{l}\text { Shale ......... } \\
\text { Shale........ }\end{array}$ & Stiff mud...... & (n) & 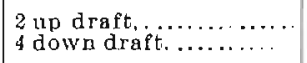 & $\begin{array}{l}\text { Stoneware, } \\
\text { Drain tile, bollow }\end{array}$ \\
\hline Fort Durite Brick and Tile Company & Fort Dodge.... & Shale... & Stiff mud. .... & Steam ........ & 2 square, 4 round down & Common brick arḍ \\
\hline Fort Dodge Clay Works. & Fort Dodge & Shale.... & Stift nud. . & Wolff \& An. & 1 continuous and 3 down & Common briels: \\
\hline Kalo Brick and Tile Company...... & Kalo ...... & Shale....... & Stiff mud.... & & 7 down draft.......... & $\begin{array}{l}\text { Comm on brick, } \\
\text { thain tile, hollow } \\
\text { blowk. }\end{array}$ \\
\hline Johnston Bros. & Kalo Junction. & Shale. & Stiff mud. & & jo down draft. ...... & $\begin{array}{l}\text { Oomm on bricks, } \\
\text { drain tile, hislow } \\
\text { bloek. }\end{array}$ \\
\hline Corey Pressed Brick Company .... & Lehigh .... & Shale ......... & Dry press .. & & $\begin{array}{l}5 \text { down draft, } 1 \text { inuffle } \\
\text { up draft. }\end{array}$ & Front briek, \\
\hline Lehigh Clay Works.. & Lehigh & Shale. & Stift mud. & & 8 down draft............ & $\begin{array}{l}\text { Drain tile, hollow } \\
\text { block }\end{array}$ \\
\hline $\begin{array}{l}\text { Jas. Campbell } \ldots \ldots \ldots \ldots \ldots \ldots \ldots \\
\text { Lehigh Brick and Tile Company... }\end{array}$ & $\begin{array}{l}\text { Lehigh .... } \\
\text { Lehigh.... }\end{array}$ & $\begin{array}{l}\text { Shale .......... } \\
\text { Shale.......... }\end{array}$ & $\begin{array}{l}\text { Stiff mud.... } \\
\text { Stiff mnd..... }\end{array}$ & & 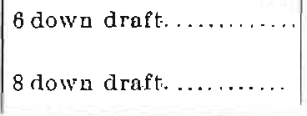 & $\begin{array}{l}\text { Comm on brick, } \\
\text { drain tile, hollow } \\
\text { block . } \\
\text { Drain tile, hollow } \\
\text { blonk. }\end{array}$ \\
\hline \multicolumn{7}{|c|}{ WINNESHIEK COUNTY. } \\
\hline $\begin{array}{l}\text { Kornmesur Bros..................... } \\
\text { Heury Schul\%. }\end{array}$ & 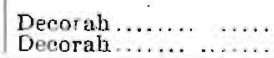 & $\begin{array}{l}\text { Surface. } \\
\text { Surface. }\end{array}$ & $\mid$ Soft mud................. & $\ldots \ldots \ldots \ldots$ & 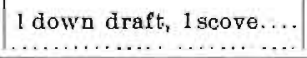 & $\begin{array}{l}\text { Common brick. } \\
\text { Gommon hrielk. }\end{array}$ \\
\hline
\end{tabular}


WOODBURY COUNTY.

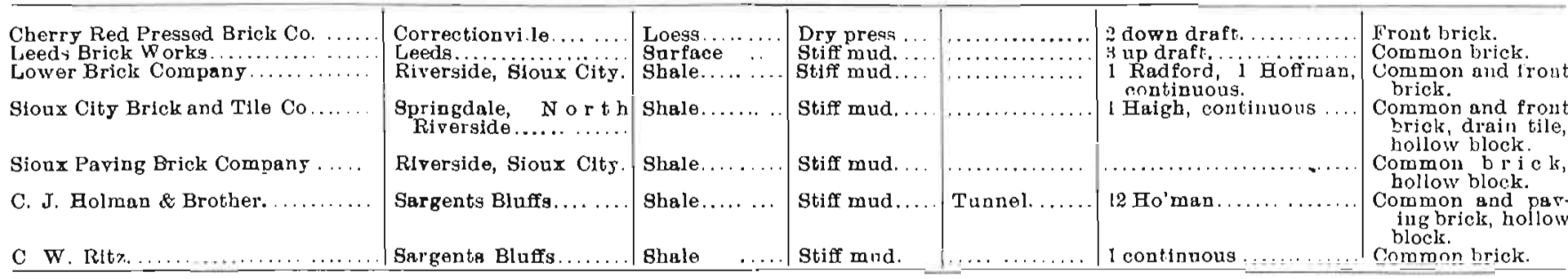

WRIGHT COUNTY.

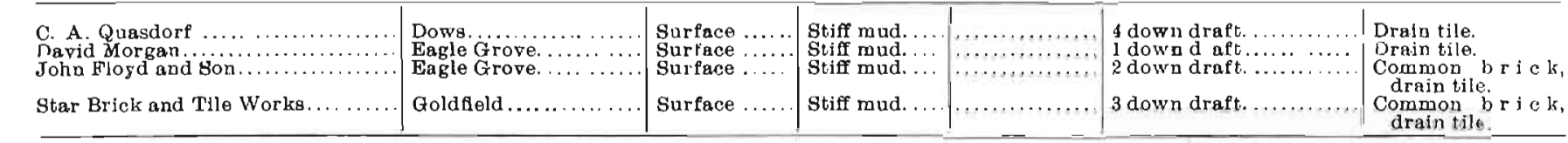




\section{ACKNOWLEDGMENTS.}

The writers of this report desire to express their appreciation and oluligation to the clay worker's of the state who have so generously co-operated in the carrying forward of the work. In fact, anything like a satisfactory report would have been impossible without their: assistance.

Mr:st of the chemical work was done by Dr. J. B. Weems, Chemist of the Survey, and the chapter entitled "Chemistry of Clays" included in this report, dealing with the methods of rational and ultimate analyse's, was jrelared by Dr. Weems, and will undoultedly prove to be a welcome contribution to cluemists generally.

The laboratory worls on the plyysical properties of clays was done by Mrr. I. A. Williams, and the chapter on teclnology bearing upon these properties, and the descriptions of clay working machinery and processes were almost wholly prejured by him. Nearly all of the cuts illustrating the various types of clay working machinery were generously loaned by the manulacturers of the most approved types in the countig, and to themi due acknowledgment is made herewith.

The chapter on "Power: Plants" prepared by Professor Bissell is timely and will undoultedly be appreciated by all progressive clay workers.

It was the intention of the writers to include an elaborate report on clay testing but owing to the press of other work Professor Marstou was unable to complete his worlk on the tests of paving brick. Fairly complete reports are submitted on common brick and dry presis brick, and synopses on hollow block and javing brick. 
In the preparation of the chapter on "The Geology of Clays" published articles and, in some instances, the unpublished notes of memburs of the Survey, to which the completeness of this chapter is largely due, were freely used and without special acknowledgment in the text.

The increasing scarcity of good lumber gives any treatise on structural materials, which may be used as substitutes for lumber, greater interest than it would otherwise deserve, and it is hoped that the present volume may be of service to contractors, buildels and prospective as well as active clay workers. 
